\title{
PDE Control of a Rotating Shear Beam with Boundary Feedback
}

\author{
Mustafa Dog̃an $^{1}$, Ömer Morgül ${ }^{2}$ \\ Department of Electrical and Electronics Engineering \\ ${ }^{1}$ Baskent University, ${ }^{2}$ Bilkent University, Turkey
}

\begin{abstract}
We consider a flexible structure modeled as a shear beam which is clamped to a rigid body at one end and is free at the other end. The whole structure is free to rotate on the horizontal plane. We first model the system by using Partial Differential Equations (PDE) and we propose boundary feedback laws to achieve set point regulation of the rotation angle as well as to suppress the elastic vibrations. The proposed control laws are based on PDE model, hence we do not resort to discretization of the system equations by available methods. We utilize a coordinate transformation based on an invertible integral transformation by using Volterra form and backstepping techniques. We also present some simulation results.

Key Words : Partial Differential Equations, Flexible Systems, Boundary Control, Shear Beam, Kernel Functions, Volterra Transformation, Backstepping.
\end{abstract}

\section{INTRODUCTION}

The progress in the construction of various mechanical structures, e.g. in robotics and space structures on macroscopic scale as well as micro-machines, atomic force microscopy etc. in microscopic scale, necessitates the use of lightweight materials for various practical reasons, see e.g. [12]. Such materials usually exhibit flexible vibrations and to model such structures one has to use Partial Differential Equations (PDE). In practice, when designing controllers for such systems, usually these PDE models are reduced to Ordinary Differential Equations (ODE) by using various methods such as model reduction, finite element analysis, discretization etc., see e.g. [3]. However, such ODE models have some drawbacks and usually the designed controllers based on these ODE models limit the performance of such systems, see e.g. [20].

One of the most frequently encountered flexible structures in mechanical structures mentioned above is the flexible beams which are typically used to model flexible links of robotic arms, tips of atomic force microscopes etc. Among the various advantages of using flexible beams, the main ones are their light weights and low energy consumptions. There are various PDE models for flexible beams such as Euler-Bernoulli, Rayleigh, Timoshenko beam equations. Among these, the most advanced and comprehensive one is the Timoshenko beam model. This model, under the

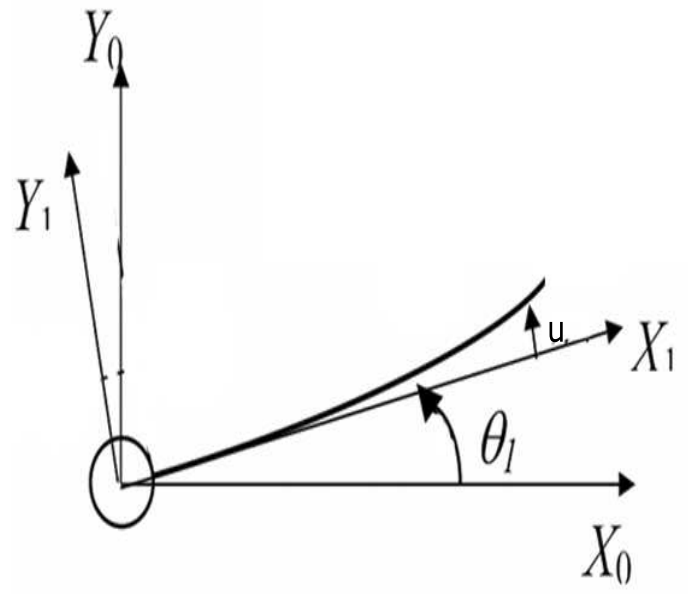

Fig. 1. Beam configuration

"slender beam" assumption, can alternatively be represented as shear beam, see e.g. [1], [2].

Various methods have been proposed for control of flexible-links in the literature, see e.g. [4], [5], [6], [7], [8], [9]. Recently, [12], [11], [10], proposed the structural approach to PDEs with backstepping techniques.

In this paper, we consider a shear beam, clamped to a rigid body in one end and is free at the other end. The whole configuration is free to rotate on the horizontal plane. We first give the equations of motion for such system. We will use PDE model for the flexible beam without resorting to reducing the resulting equations to an ODE model. Our control objective is to rotate the flexible beam to a desired angle and suppress the flexural vibrations. We use a technique that is first introduced in [12], [11], [10] to transform the system equations to another set of equations which has well-known stability properties. This transformation is done by using an invertible integral operator (Volterra type) with a smooth kernel. We give the analytical expression of such a kernel. In the process of obtaining an appropriate kernel function, we also obtain appropriate boundary control law to stabilize the closed loop system. We also present some simulation results which confirms the stability of the closed loop system. Finally we give some concluding remarks. 


\section{Analytical Model}

We consider a flexible structure consisting of a flexible (shear) beam which is clamped to a rigid body at one end, free at the other and as shown in Figure 1. Referring to Figure 1 the various symbols represent the following; $X_{o} Y_{o}$ : global inertial system of coordinates; $X_{1} Y_{1}$ : bodyfixed system of coordinates attached to undeformed beam, $\theta_{1}$ : angular displacement of beam, $u$ : flexural displacement of beam.

In this section, starting with Timoshenko beam model, the partial differential equations with boundary conditions are derived using the Volterra state transformation, see e.g. [12], [14]. The link is modelled in clamped-free configuration, since natural modes of the separated clamped-free links agree very well with actual ones compared to pinnedfree configuration, see e.g. [15]. Assuming the manipulator rotates in the horizontal plane, in the absence of gravity the potential energy depends only on the flexural deflections.

\begin{tabular}{|l|l|}
\hline Parameter & Description \\
\hline$E$ & Young's Modulus \\
\hline$G$ & Shear Modulus \\
\hline$A$ & cross-sectional area \\
\hline$I$ & cross-sectional area moment \\
\hline$I_{h}$ & Inertia of the hub \\
\hline$L$ & Length of the beam \\
\hline$x$ & Coordinate along the axial center \\
\hline$u(x, t)$ & Transverse movement \\
\hline$\alpha(x, t)$ & Angle of distortion due to shear \\
\hline$\dot{u}(x, t)$ & Time rate of transverse movement \\
\hline$u_{x}(x, t)$ & Axial rate of transverse movement \\
\hline$\rho$ & Linear density \\
\hline$\tau_{1}$ & Input torque at base motor \\
\hline$\theta_{1}$ & Angular position of the beam due to rotation \\
\hline
\end{tabular}

TABLE I

PARAMETERS FOR PDE MODEL.

The equations of motion for Timoshenko beam can be given with the notation in Table I as follows

$$
\begin{gathered}
\epsilon \ddot{u}=b u_{x x}-\alpha_{x}-\epsilon x \ddot{\theta}_{1} \\
\mu \epsilon \ddot{\alpha}=\epsilon \alpha_{x x}-\alpha+b u_{x}
\end{gathered}
$$

where a dot represents time derivative, a subscript as in $u_{x}$ denotes spatial derivative with respect to (w.r.t.) $x$, for $0 \leq x \leq L$ and $t \geq 0$. Here, $E I$ denotes bending stiffness, $b$ is defined as $b=E I / \rho, E A$ denotes axial stiffness, $\mu$ is defined as $\mu=\rho / E A$ and shear coefficient, $\epsilon$ is a linear function of $E I / G A$, see e.g. [16], [17]. Since the ratio between the length of the beam and its thickness is sufficiently large, we can take $\mu=0$ approximately. Then, the slender beam can easily be modelled as shear beam, see e.g. [1], [16]. Thus, the governing equations for a rotating shear beam can be given below

$$
\begin{array}{r}
\epsilon \ddot{u}=b u_{x x}-\alpha_{x}-\epsilon x \ddot{\theta}_{1} \\
0=\epsilon \alpha_{x x}-\alpha+b u_{x}
\end{array}
$$

By differentiating ( 3) w.r.t. $x$ and add to (4), we obtain

$$
\epsilon \ddot{u}_{x}-b u_{x x x}+\alpha_{x x}+\epsilon \ddot{\theta}_{1}+\epsilon \alpha_{x x}-\alpha+b u_{x}=0
$$

now if we differentiate (5) w.r.t. $x$ and subtract $1 / \epsilon$ times (3) then we have

$$
\begin{gathered}
\epsilon \ddot{u}_{x x}-b u_{x x x x}+(1+\epsilon) \alpha_{x x x}+b u_{x x} \\
-\alpha_{x}-\ddot{u}+\frac{b}{\epsilon} u_{x x}-\frac{1}{\epsilon} \alpha_{x}-x \ddot{\theta}_{1}=0 .
\end{gathered}
$$

Finally by calculation of $\alpha_{x x x}$ from (4), we obtain the following shear beam model as single second-order-in-time, fourth-order-in-space PDE

$$
\ddot{u}-\epsilon \ddot{u}_{x x}+b u_{x x x x}=-x \ddot{\theta}_{1}
$$

The usual clamped-free boundary conditions for the shear beam can be given as follows, see e.g. [18]

$$
\begin{aligned}
& u(0, t)=0 \\
& u_{x}(0, t)=\alpha(0, t) \\
& \alpha(L, t)=0 \\
& u_{x x}(L, t)=0
\end{aligned}
$$

By using (4), we can obtain $\alpha(x, t)$ in terms of $u$. By using Laplace transform in (4), we get the following after some calculations and dropping the $t$ argument in the related functions, see e.g. [12]

$$
\alpha_{x}(x)=-a^{2} c \int_{0}^{x} \sinh (c x-c y) u(y) d y-a^{2} u(x)
$$

where $a^{2}=b / \epsilon$ and $c^{2}=1 / \epsilon$. By substituting ( 10) into (3), governing equations ( $3-4$ ) can be simplified as single equation below. For the rigid body rotation, by applying conservation of momentum at the base, we obtain openloop system as follows

$$
\begin{array}{r}
\epsilon \ddot{u}=b u_{x x}+a^{2} u+a^{2} c \int_{0}^{x} \sinh (c x-c y) u(y) d y \\
-\epsilon x \ddot{\theta}_{1} \\
I_{h} \ddot{\theta}_{1}-E I u_{x x}(0, t)=\tau_{1}
\end{array}
$$

\section{Controller Design}

The controller for the rigid part can be designed to provide exponential decaying of derivatives and to achieve the desired set point such that

$$
\tau_{1}=-E I u_{x x}(0, t)-k_{1} \dot{\theta}_{1}-k_{2}\left(\theta_{1}-\theta_{d}\right)
$$

where $k_{1}, k_{2}$ are positive constants and $\theta_{d}$ are the desired position. To simplify the flexible equation (11), we first define a new state variable $w(\cdot)$ by applying an invertible Volterra state transformation with smooth kernel $k(x, y)$

$$
w(x)=u(x)-\int_{0}^{x} k(x, y) u(y) d y
$$


For the rationale and methodology behind using such a transformation, see e.g. [12], [14]. The kernel $k(x, y)$ should be chosen so that the resulting equations in terms of the transformed variable $w(x)$, has nice stability properties. Such a resulting system can be given as follows

$$
\begin{aligned}
\epsilon \ddot{w} & =b\left(w_{x x}-e w\right) \\
w_{x}(L) & =-c_{o} \dot{w}(L) \\
w_{x}(0) & =c_{1}(\epsilon / b) \ddot{\theta}_{1}
\end{aligned}
$$

where $e, c_{o}, c_{1}$ are positive controller gains. Note that the boundary condition ( 17 ) is of crucial importance to obtain equations (10) and (11), see also remark 3.2. Hence, the open-loop system given by (11-12) can be transformed into the closed-loop system given by $(15-17)$ with the following boundary control law

$$
\begin{aligned}
u_{x}(L, t)= & \int_{0}^{L} k_{x}(L, y) u(y) d y+k(L, L) u(L, t) \\
& -c_{o} \dot{u}(L, t)+c_{o} \int_{0}^{L} k(L, y) \dot{u}(y) d y .
\end{aligned}
$$

Obviously we need to describe the kernel $k(x, y)$ or its properties at this stage. In addition to equations (11-17), the following equations are also required to obtain the control law (18) and some conditions for the kernel:

$$
\begin{aligned}
w_{x}(x) & =u_{x}(x)-\int_{0}^{x} k_{x}(x, y) u(y) d y-u(x) k(x, x) \\
w_{x x}(x) & =u_{x x}(x)-u(x)\left[k_{x}(x, x)+k_{y}(x, x)\right] \\
& -u_{x}(x) k(x, x)-u(x) k_{x}(x, x) \\
& -\int_{0}^{x} k_{x x}(x, y) u(y) d y \\
\epsilon \ddot{w}(x) & =\epsilon \ddot{u}(x)-\int_{0}^{x} k(x, y) \epsilon \ddot{u}(y) d y
\end{aligned}
$$

By following the methodology introduced in [12], the term, $\epsilon \ddot{u}(\cdot)$ should be substituted in (20) by using (11). Thus, obtained $\epsilon \ddot{w}(x)$ and $w_{x x}(x)$ given by (19) can be replaced in (15). After some calculations, it can be shown that the required kernel $k(x, y)$ should satisfy the non-homogenous PDE as follows

$$
\begin{aligned}
k_{y y}-k_{x x}+f k & =\epsilon^{-1.5} \sinh (c x-c y) \\
k(x, x) & =-f x / 2 \\
k(0,0) & =0 \\
k(x, 0) & =x-\int_{0}^{x} y k(x, y) d y \\
k_{y}(x, 0) & =0
\end{aligned}
$$

where $f=a^{2} / b+e$. The analytical solution of ( 21$25)$ for the kernel function $k(x, y)$ can be obtained by using standard methods see e.g. [19]. After some lengthy calculations, we obtain the following explicit form of the kernel $k(x, y)$ :

$$
\begin{aligned}
& k(x, y)=0.5[h(0)+h(L)]+0.5 \int_{0}^{L} J_{0}(z) g(\xi) d \xi \\
& -0.5 y f \int_{0}^{L}\left(J_{1}(z) / z\right) h(\xi) d \xi-m \sinh (c x-c y)
\end{aligned}
$$

where $m=-\epsilon^{-1.5} / f$, and $z=\sqrt{\left(y^{2}-(x-\xi)^{2}\right) f}$, $J_{0}(\cdot), J_{1}(\cdot)$ are Bessel functions, $h(x)=(m-$ $0.81) \sinh (c x)+0.62521 x$, and $g(x)=-c m \cosh (c x)$. Note that the functions $h(\cdot)$ and $g(\cdot)$ are generated by boundary conditions of the kernel. For an alternate expression of the kernel, see the Appendix.

Due to space limitations, we do not present any stability results at the moment. But note that the stability of closed loop system can be analyzed with the Lyapunov stability theory by using the following Lyapunov function candidate [8]

$$
V=d_{1}\left(\left\|w_{x}\right\|^{2}+e\|w\|^{2}\right)+\epsilon\|\dot{w}\|^{2}+d_{2} \epsilon\langle w, \dot{w}\rangle
$$

where $d_{1}, d_{2}$ appropriate positive constants, $\left\|w_{x}\right\|^{2}=$ $\int_{0}^{L} w_{x}^{2} d x$ is the 2 -norm and $<\cdot>$ denotes the usual inner product in $L_{2}(0, L)$ space.

Remark 3.1: Note that to implement the control law given by (18), we need the measurements of $u(x, t), u(L, t)$ and $\dot{u}(L, t)$. The last two can be measured easily. However, to measure $u(x, t)$ is not practical, but it can be estimated by using an appropriate observer. Following the ideas given in [12], such an observer can be designed with the help of the kernel $k(x, y)$ given above as explained in the next section.

Remark 3.2: The boundary condition (17) can be implemented as the secondary controller easily due to actuation and measurement at the clamped-end. Furthermore, this secondary control law is indispensable to simplify the open loop system and to obtain the analytic kernel solution. This is achieved by decoupling the rigid and flexible coordinates, result in the boundary condition ( 8) will decay exponentially fast enough. The whole structure of the proposed design and its implementation can be straightforward in a pure control way, compare with the much more complex handling of the same problem, see e.g. [13].

\section{OBSERVER DESIGN}

Similar to the controller design, to define the observer error dynamics, we need an auxiliary dynamics with wellknown stability properties. Such a system can be given as follows

$$
\begin{aligned}
\epsilon \ddot{\tilde{w}} & =b\left(\tilde{w}_{x x}-e_{2} \tilde{w}\right) \\
\tilde{w}_{x}(L) & =-c_{2} \dot{\tilde{w}}(L) \\
\tilde{w}_{x}(0) & =0
\end{aligned}
$$


where $e_{2}, c_{2}$ are positive observer gains and observer error assumed to be zero at clamped end. After applying Volterra state transformation, observer error, $\tilde{u}(x)$ can be expressed as follows

$$
\tilde{u}(x)=\tilde{w}(x)-\int_{0}^{x} p(x, y) \tilde{w}(y) d y .
$$

Also, observer error can be defined as $\tilde{u}(x)=u(x)-\hat{u}(x)$ here and observer kernel, $p(x, y)$ is the dual version of the controller kernel $k(x, y)$ with appropriate boundary conditions. Hence, we are ready to introduce observer dynamics which is driven by $\tilde{w}(x)$ such that

$$
\begin{array}{r}
\epsilon \ddot{\hat{u}}=b \hat{u}_{x x}+a^{2} \hat{u}+a^{2} c \int_{0}^{x} \sinh (c x-c y) \hat{u}(y) d y \\
-\epsilon x \ddot{\theta}_{1}+p_{y}(x, L)[u(L, t)-\hat{u}(L, t)] \\
-c_{2} p(x, L)[\dot{u}(L, t)-\dot{\hat{u}}(L, t)] .
\end{array}
$$

Note that $p_{y}(x, L), p(x, L)$ are dual counterparts to the controller gains $k_{x}(L, y), k(L, y)$. The observer dynamics comply with usual observer setup which can be explained as copy of the plant plus error feedback. After some lengthy calculations by using the integral transformation (31) and auxiliary dynamics ( 28 ), we get the following equations

$$
\begin{array}{r}
p_{x x}-p_{y y}+\tilde{f} p=\epsilon^{-1.5} \sinh (c x-c y) \\
a^{2} c \int_{0}^{x} \sinh (c x-c y) \int_{0}^{y} p(y, s) \tilde{w}(s) d s d y= \\
c_{2} p(x, L) \dot{\tilde{u}}(L, t)-p_{y}(x, L) \tilde{u}(L, t)
\end{array}
$$

where $\tilde{f}=a^{2} / b+e_{2}$. The analytical solution of (33) for the observer kernel function $p(x, y)$ can be obtained easily by duality of equations ( 21-33). Finally, equation (34) will help us to construct the observer dynamics with auxiliary one, $\tilde{w}(x)$ in lieu of $\tilde{u}(x)$. Besides, the new control law based on observer can be given below

$$
\begin{aligned}
u_{x}(L, t)= & \int_{0}^{L} k_{x}(L, y) \hat{u}(y) d y+k(L, L) u(L, t) \\
& -c_{o} \dot{u}(L, t)+c_{o} \int_{0}^{L} k(L, y) \dot{\hat{u}}(y) d y .
\end{aligned}
$$

\section{Simulation Results}

For the simulations, we will use the following set of parameters which are taken from [20]:

The proposed control scheme is tested with the simulation program implemented in MATLAB. The PDEs are discretized in the space domain by the finite difference method, to obtain ODEs at each of the nodes. Then, ODEs are solved numerically. Instead of dealing with complexity of the fourth order derivative approximation, the second order derivative approximation has been used by the virtue of coordinate transformation. Those states are

\begin{tabular}{|l|l|}
\hline Parameter & Value \\
\hline Length of the beam, & $L=0.6 \mathrm{~m}$ \\
\hline Time step & $\Delta t=1 e-4 \mathrm{sec}$ \\
\hline Spatial step & $\Delta x=L / 30$ \\
\hline Young's Modulus, $E$ & $70 \quad \mathrm{GPa}$ \\
\hline Density & $2742 \quad \mathrm{kgm}^{-3}$ \\
\hline Thickness of the beam $(\mathrm{m})$ & $t_{1}=0.003175$ \\
\hline Height of the beam & $b_{o}=0.0654 \mathrm{~m}$ \\
\hline Shear coefficient & $\epsilon=1.6801$ \\
\hline Hub inertia $\left(\mathrm{kgm}^{2}\right)$ & $I_{h}=0.0055$ \\
\hline$\theta_{d}($ desired) & $\pi / 3 \quad \mathrm{rad}$ \\
\hline Controller gains & $c_{o}=59$ \\
\hline$k_{1}=I_{h} \cdot 600, \quad k_{2}=I_{h} \cdot 800$ & $c_{1}=1, e=0.9$ \\
\hline Observer gains & $c_{2}=82$ and $e_{2}=0.3$ \\
\hline
\end{tabular}

TABLE II

PARAMETERS OF THE BEAM.

more meaningful in a real problem as well since they corresponds to physical variables such as deflections, velocity and bending moments. However, the number of ODEs to solve and the computation time are increased in return of the robust stability of the numeric scheme. The explicit finite difference scheme that requires very small time-steps and easy to implement efficiently, is adapted from [21]. Chaotic vibrations of a modified Euler-Bernoulli beam that are difficult to catch, have been solved successfully by the same numeric scheme in [21]. On the other hand, robust numerical stability is succeeded by making the ratio $\Delta t / \Delta x^{2} \leq 0.5$ as low as possible. The parameters used in the model for system ( 11 - 12), are listed in Table II. The simulation results are presented in Figures 2 - 7. Note that Figure 4 represents the bending strain of the beam near the free end point $x=L$. Decoupling between the rigid coordinates and flexible ones is succeeded by control laws, and is observed during simulations. For $\theta_{1}$ and $\dot{\theta}_{1}$, see Figures 5 - 6, converging properties (two exponential decaying modes for derivatives) can be adjusted independently with $k_{1}, k_{2}$. Therefore, the $\ddot{\theta}_{1}$ can be used at boundary condition ( 17) by exponential decaying time history, and also to simplify the closed-loop system. Fast convergence to zero for observer error rate is quite satisfactory in Figure 8. Finally, smooth time histories of all variables of interest without overshoot show the effectiveness of the controller performance with relatively low control energy.

\section{CONCLUSIONS}

The most complex beam model is simplified and became easy to analyze and to control. Also, we do not use any damping term in the system model. In this paper, we consider the set point control of a rotating shear beam. We assumed that the shear beam is free to rotate on the horizontal plane. In this research, the system equations is first transformed into another set of equations which has well-known stability properties by using an invertible Volterra state transformation. We also obtained the kernel 


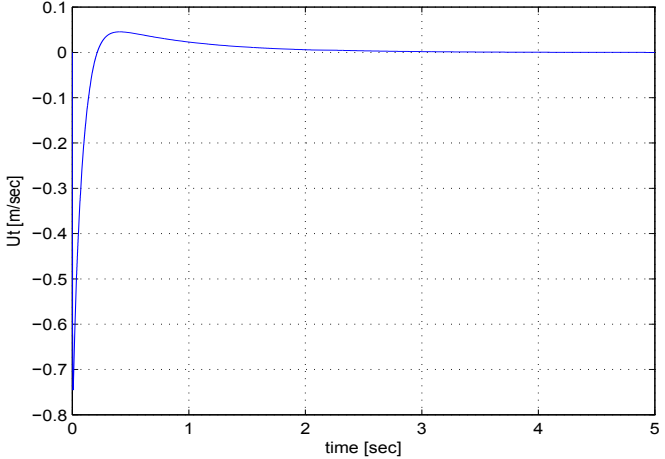

Fig. 2. Flexural Velocity at the end of the beam

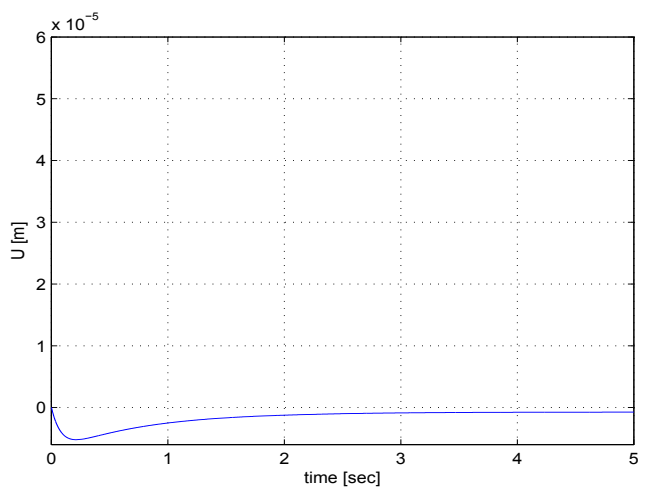

Fig. 3. Deflection at the end of the beam

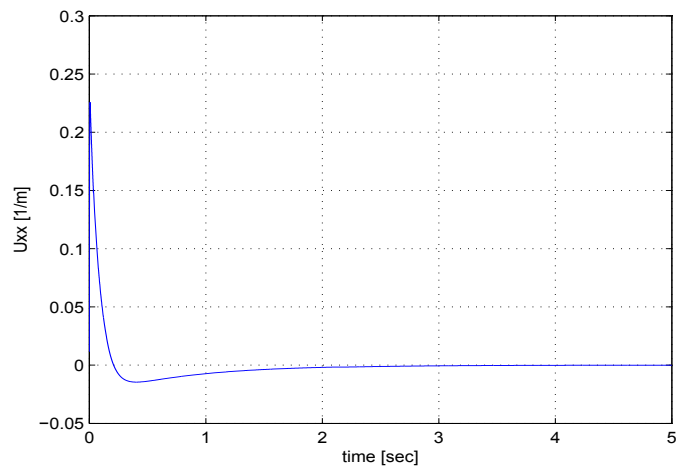

Fig. 4. Bending strain of the beam

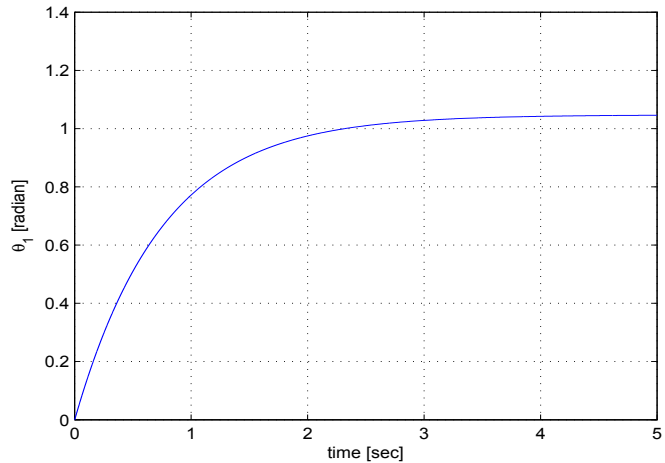

Fig. 5. Joint Angle of the beam

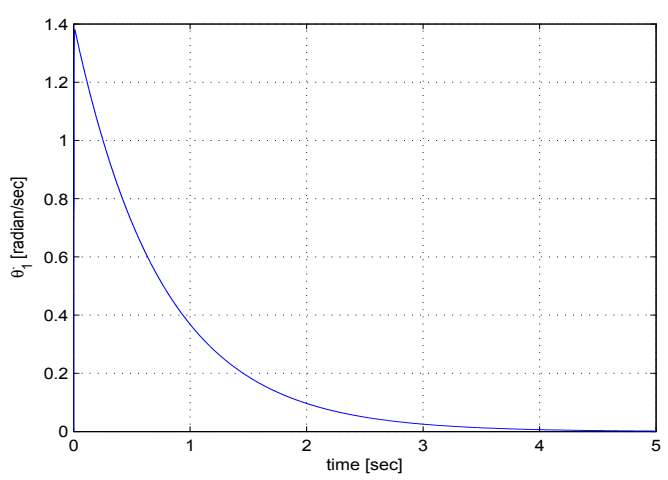

Fig. 6. Joint Angular Velocity of the beam

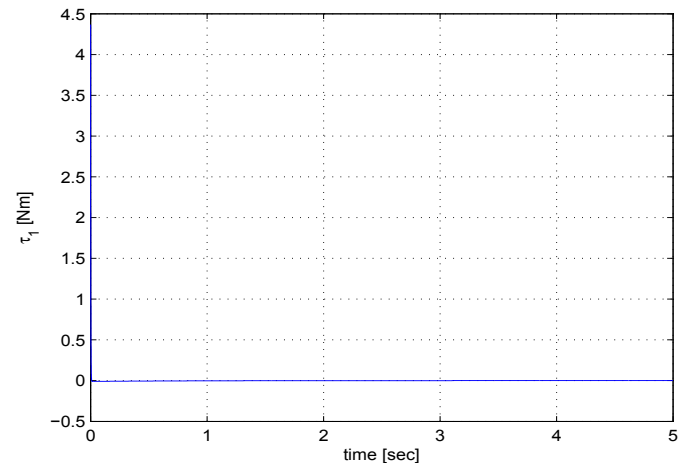

Fig. 7. Control Torque 


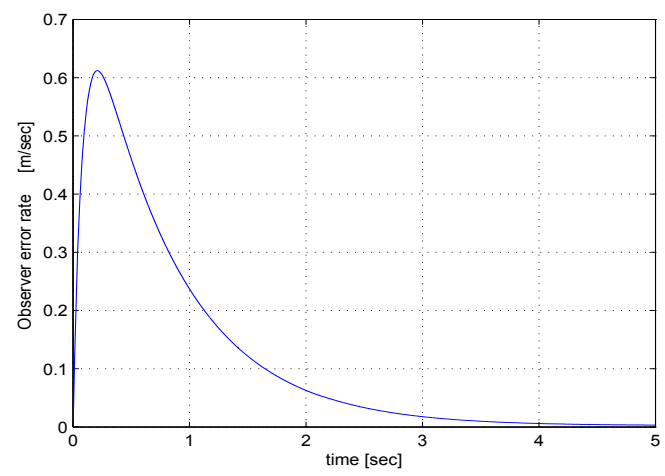

Fig. 8. Observer Error Rate

function of such a transformation analytically by the virtue of proposed design. This process is also very efficient to improve the observer dynamics that will give the required boundary control laws. Our simulation results show that the proposed control scheme is effective in both achieving correct orientation and in suppression of flexural vibrations.

\section{APPENDIX}

Note that the kernel expression given by (26) is obtained by changing variables to have homogenous hyperbolic kernel PDE in lieu of ( 21) with appropriate boundary conditions as follows

$$
\begin{aligned}
r(x, y) & =k(x, y)+m \sinh (c x-c y) \\
r_{y y}-r_{x x}+f r & =0 \\
r(x, x) & =-f x / 2 \\
r(0,0) & =0 \\
r(x, 0) & =k(x, 0)+m \sinh (c x) \\
r_{y}(x, 0) & =-c \cdot m \cosh (c x)
\end{aligned}
$$

where $m=-\epsilon^{-1.5} / f$, and the terms that produce by the second order spatial derivatives, cancelled each other. By performing integrals in (26) that contains Bessel functions $J_{0}(\cdot)$ and $J_{1}(\cdot)$, and after some lengthy calculations, we obtain the following explicit form for the kernel $k(x, y)$ :

$$
\begin{array}{r}
k(x, y)=0.5[h(0)+h(L)] \\
+(c . m / 4)(\sinh (c x)+\cosh (c x)) \\
\cdot \frac{1}{\sqrt{c^{2}+f}} \exp \left(-y \sqrt{c^{2}+f}\right) \\
+\frac{1}{4}(\sinh (c x)+\cosh (c x)) \\
.(m-0.81039)\left[\exp \left(-y \sqrt{c^{2}+f}\right)-\exp (-c y)\right]
\end{array}
$$

$$
\begin{array}{r}
-0.62521 x \frac{(1-\cos (y \sqrt{f}))}{2(m-0.81039)} \\
+\frac{0.62521 f y}{2(m-0.81039)}\left[1-J_{0}\left(\sqrt{\left(y^{2}-x^{2}\right) f}\right)\right] \\
-m \sinh (c x-c y)
\end{array}
$$

\section{REFERENCES}

[1] L. Meirovitch, Fundamentals of Vibrations, New York: McGraw-Hill, 2001.

[2] H. Baruh, Analytical Dynamics, Boston: McGraw-Hill, 1999.

[3] M. Dog̃an, Y. Istefanopulos, 'Optimal Nonlinear Controller Design for Flexible Robot Manipulators with Adaptive Internal Model', IET Control Theory and Applications, 1, 3, (2007), 770-778.

[4] J.U. Kim and Y. Renardy, 'Boundary control of the Timoshenko beam', SIAM J. of Control and Optimization, vol. 25, pp. 1417-1429, 1987.

[5] F.Y. Wang and Y. Gao , Advanced Studies of Flexible Robotic Manipulators, New Jersey: World Scientific, 2003.

[6] Z. H. Luo, B. Z. Guo and Ö. Morgül, Stability and Stabilization of Infinite Dimensional Systems with Applications, London: SpringerVerlag, 1999.

[7] Ö. Morgül, 'Orientation and Stabilization of a Flexible Beam Attached to a Rigid Body: Planar Motion', IEEE Transactions on Automatic Control, vol.36, No.8, pp.953-962, 1991.

[8] Ö. Morgül, 'Dynamic boundary control of the Timoshenko beam', Automatica, vol.28, No.6, pp.1255-1260, 1992.

[9] B. Z. Guo, 'Riesz Basis Property and Exponential Stability of Controlled Euler-Bernoulli Beam Equations with Variable Coefficients', SIAM J. of Control and Optimization, vol.40, No.6, pp.1905-1923, 2002.

[10] A. Smyshlyaev and M. Krstic, 'Closed-form boundary state feedbacks for a class of 1-D partial integro-differential equations', IEEE Transactions on Automatic Control, vol.49, No.12, pp.2185-2202, 2004.

[11] A. Smyshlyaev and M. Krstic, 'Backstepping observers for a class of parabolic PDEs', Systems and Control Letters, vol.54, pp.613-625, 2005.

[12] M. Krstic, A. A. Siranosian, and A. Smyshlyaev , 'Backstepping boundary controllers and observers for the slender Timoshenko beam:Part I-Design', Proceedings of the American Control Conference, pp. 2412-2417, Minneapolis, 2006.

[13] M. Krstic, B. Z. Guo, A. Balogh, and A. Smyshlyaev , 'Control of a tip-force destabilized shear beam by observer-based boundary feedback', SIAM J. of Control and Optimization, vol.47, No.2, pp.553-574, 2008.

[14] D. Porter and D.S.G. Stirling, Integral Equations, Cambridge: Cambridge University Press, 1990.

[15] G.G. Hastings and W.J.Book,'A Linear Dynamic Model for Flexible Robotic Manipulators',IEEE Control System Magazine, vol.7, pp.6164, 1987.

[16] J.N. Reddy, An Introduction to the Finite Element Method, New York: McGraw-Hill, 1993.

[17] L. Sievers, M. J. Balas, A. V. Flotow 'Modelling of Web conveyance systems for multi-variable control', IEEE Transactions on Automatic Control, vol.33, No.6, pp.524-531, 1988.

[18] Meirovitch, L., Analytical Methods in Vibrations, London:MacMillan, 1967.

[19] L. Debnath, Nonlinear PDEs for scientists and engineers, Boston: Birkhauser, 1997.

[20] M. Dog̃an, 'Optimal Nonlinear Controller Design for Flexible Robot Manipulators', Ph.D. Thesis, Bog̃aziçi University, Istanbul, Jan. 2006.

[21] N.S. Abhyankar, E.K. Hall II, S.V. Hanagud, 'Chaotic Vibrations of Beams:Numerical Solution of Partial Differential Equations', $A S M E$ Journal of Applied Mechanics, vol.60, pp.167-174, 1993. 